\title{
INTERPRETIVE SUMMARIES, FEBRUARY 2014
}

Risk-based audit selection of dairy farms. By van Asseldonk and Velthuis, page 592. To select higher-risk farms for an audit, we tested the relation between the outcome of farm audits and bulk milk quality tests before the audit. The analysis comprised 28,358 farm audits and all conducted quality tests of bulk milk samples 12 months before the audit. Milk quality information can be used to select higher-risk farms to be audited more frequently. In order to capture 25, 50 , and $75 \%$ of the farm population with poor process standards (i.e., rejected), only 8,20 , and $47 \%$ of the population, respectively, had to be sampled based on a risk-based selection approach.

http://dx.doi.org/10.3168/jds.2013-6604.

Assessing the yield, microstructure, and texture properties of miniature Chihuahua-type cheese manufactured with a phospholipase $\mathbf{A}_{1}$ and exopolysaccharide-producing bacteria. By Trancoso-Reyes et al., page 598. Cheese yield is a crucial determinant of profitability in cheese manufacturing plants; therefore, different methods have been developed to increase yield. In this work, we used an enzyme that modifies milk fat combined with a bacterial culture that produces gums as a means to improve the yield of Chihuahua cheese. Incorporation of the enzyme together with the use of the bacterial culture increased the yield in the manufacture of Chihuahua cheese from 9.21 to $10.81 \mathrm{~kg}$ of cheese $/ 100 \mathrm{~kg}$ of milk, without changing the perception of texture or flavor. http://dx.doi.org/10.3168/jds.2013-6624.

Identification of a microscopically selected microorganism in milk samples. By Bracke et al., page 609. Identification of microscopically observed microbial contaminants in food products is hindered by several challenges, including (1) the low quantity of contaminating organisms in a complex matrix with abundant other organisms, and (2) the difficulty of culturing the contaminating microorganism due to changes in its morphology (e.g., spores). Consequently, it is very difficult to obtain sufficient quantities of these contaminating microorganisms, allowing identification only by biochemical assays or profiling methods. We developed a selective identification method based on laser capture microdissection and succesfully applied our method in dairy samples.

http://dx.doi.org/10.3168/jds.2013-6932.

Oxidative stability of yogurt with added lutein dye. By Domingos et al., page 616. Exposure of yogurts to light in supermarkets favors oxidation due to the presence of riboflavin. This compound is capable of absorbing light, unleashing reactions responsible for off-flavor formation and the loss of nutrients. The addi- tion of lutein to yogurts could prevent the photooxidative damage by acting as a light filter and scavenging reactive species. In addition, lutein has been associated with the reduction and prevention of age-related macular degeneration. The objective of the present work was to evaluate the influence of adding lutein dye on the oxidative stability of yogurt.

http://dx.doi.org/10.3168/jds.2013-6971.

Volatile organic compounds profile during milk fermentation by Lactobacillus pentosus and correlations between volatile flavor and carbohydrate metabolism. By Pan et al., page 624. The naturalness and agreeable taste of yogurt make it an attractive food for consumption. Flavor, as one of the most important taste properties determining the acceptability and preference of fermented milks, is influenced by compositional and processing factors. We explored the metabolism of lactose and xylose by Lactobacillus pentosus and found that xylose metabolized by $L b$. pentosus strongly affects the flavor of yogurt. Production of volatile flavor components, such as 2,3-butanedione and acetic acid, is important in yogurt production. Xylose metabolized by $L b$. pentosus has potential value in the dairy industry, particularly in the consumer acceptability of fermented milk products.

http://dx.doi.org/10.3168/jds.2013-7131.

Thermal inactivation kinetics of Shiga toxinproducing Escherichia coli in buffalo Mozzarella curd. By Trevisani et al., page 642. Use of raw milk is allowed in the production of Mozzarella di bufala Campana because the curd is spun and stretched in hot water at 85 to $90^{\circ} \mathrm{C}$, thus reaching temperatures between $65^{\circ} \mathrm{C}$ and $80^{\circ} \mathrm{C}$. Shiga toxin-producing Escherichia coli (STEC) may be present on buffalo dairy farms and in raw milk, so challenge tests are useful to predict the rate of inactivation of STEC during heat treatments. A 4-log reduction of STEC (serogroups O26 or O157) was achieved when curd was heated at $68^{\circ} \mathrm{C}$ for 2.6 to 6.3 min or at $80^{\circ} \mathrm{C}$ for 2.1 to $2.3 \mathrm{~min}$. Thermal inactivation models were developed to provide quantitative estimations of the microbial reduction to be further used for risk assessment.

http://dx.doi.org/10.3168/jds.2013-7150.

Oral administration of bovine lactoferrin attenuates ultraviolet $\mathrm{B}$-induced skin photodamage in hairless mice. By Murata et al., page 651. Lactoferrin is recognized as a host defensive glycoprotein, especially for newborn infants; however, the effects of lactoferrin on ultraviolet (UV)-induced skin photodamage have not been elucidated. In this report, we describe for the first time that oral supplementation with lactoferrin has a protective effect against UV-induced skin aging, includ- 
ing morphological changes and skin-barrier damage, in hairless mice. Our findings suggest that oral supplementation with lactoferrin exerts an antiinflammatory activity by inhibition of UV-stimulated interleukin-1 $\beta$ production and prevents epidermal barrier dysfunction. http://dx.doi.org/10.3168/jds.2013-7153.

Effect of moderate inlet temperatures in ultrahigh-pressure homogenization treatments on physicochemical and sensory characteristics of milk. By Amador-Espejo et al., page 659. Cooked off-flavor in ultra-high-temperature (UHT) milk is the greatest disadvantage of this treatment. Ultra-highpressure homogenization (UHPH) is a novel process to treat milk that can achieve the same degree of microbial inactivation as heat treatment, but with fewer changes in sensory and physicochemical characteristics. The objective of this project was to evaluate the effect of UHPH at moderate temperatures on the physicochemical and sensory characteristics of milk. Sterility in UHPH milk can be obtained, with a less intense cooked off-flavor and similar or better physicochemical characteristics compared with UHT milk.

http://dx.doi.org/10.3168/jds.2013-7245.

Antiproliferative activity of tea catechins associated with casein micelles, using HT29 colon cancer cells. By Haratifar et al., page 672. Tea polyphenols have been reported to exhibit several beneficial health effects. However, to exert their benefits in vivo, polyphenols must be available and active. We hypothesized that due to the binding of milk proteins with epigallocatechin gallate (EGCG), milk could encapsulate and deliver this bioactive molecule. Nanoencapsulated EGCG was able to decrease the proliferation of HT-29 cancer cells, demonstrating that bioavailability may not be reduced by EGCG-protein interactions. Therefore, milk is an ideal platform for delivery of bioactive compounds to obtain a new generation of dairy products that provide additional benefits to human health.

http://dx.doi.org/10.3168/jds.2013-7263.

Effect of colostrum on gravity separation of milk somatic cells in skim milk. By Geer and Barbano, page $68 \%$. We determined that milk somatic cells (SC) separate due to gravity in raw and minimally pasteurized raw milk, but at higher pasteurization temperatures, subsequent gravity separation of SC does not occur. Addition of colostrum (a good source of immunoglobulins) restored the gravity separation of $\mathrm{SC}$ in skim milk that was heated to high temperature. Immunoglobulins may also play a role in the gravity separation of bacteria and spores in skim milk.

http://dx.doi.org/10.3168/jds.2013-7360.

Production of galactooligosaccharides using a hyperthermophilic $\beta$-galactosidase in permeabi- lized whole cells of Lactococcus lactis. By Yu and O'Sullivan, page 694. Galactooligosaccharides (GOS) can be produced from lactose by $\beta$-galactosidase, but the process is more efficient at high temperatures. A synthetic gene (lacSt) encoding a hyperthermophilic $\beta$-galactosidase was constructed and introduced into Lactococcus lactis, which enabled production of Lc. lactis cells containing high levels of this enzyme. Following permeabilization of these whole cells with ethanol, more than $197 \mathrm{~g} / \mathrm{L}$ of GOS was obtained from $40 \%$ initial lactose. However, the highest conversion rate was found for a $5 \%$ lactose solution, suggesting future application for the production of highly GOS-enriched foods from whey-based substrates using this cost-effective method. http://dx.doi.org/10.3168/jds.2013-7492.

Short communication: Genetic characterization of antimicrobial resistance in Acinetobacter isolates recovered from bulk tank milk. By Tamang et al., page 704. The nonfermenting bacteria Acinetobacter have been previously reported as one of the agents of bovine mastitis. In this study, we investigated the genetic basis of antimicrobial resistance in Acinetobacter isolates recovered from bulk tank milk samples in Korea. Understanding the resistance mechanism associated with the resistance phenotype and the genes involved in resistance may help in the development of strategies to control infections such as mastitis and to prevent further dissemination of antibiotic resistance genes. To the best of our knowledge, this is the first report of molecular characterization of antimicrobialresistant Acinetobacter spp. from milk. http://dx.doi.org/10.3168/jds.2013-7403.

Technical note: The equivalency of sodium results in cheese digested by either dry ashing or microwave-accelerated digestion. By Schoenfuss et al., page 710. Traditional sample preparation of cheese before mineral analysis by atomic absorption spectroscopy requires wet ashing with nitric acid or dry ashing, both of which can be time consuming and hazardous. A microwave-accelerated reaction system allows for rapid and automatic wet acid digestions of multiple samples in less than $1 \mathrm{~h}$. Samples of blue cheese (138, in duplicate) were prepared for atomic absorption spectroscopy by both dry ashing and microwave-accelerated digestion, and the sodium values obtained were compared. The microwave method was found to produce results equivalent to those achieved by the dry-ashing procedure.

http://dx.doi.org/10.3168/jds.2013-7420.

Exploring the value of routinely collected herd data for estimating dairy cattle welfare. $B y$ de Vries et al., page 715. Routine on-farm assessment of dairy cattle welfare is time consuming and, therefore, expensive. Our aim was to explore the value of routine 
herd data available in national databases for estimating dairy cattle welfare at the herd level. We found that the accuracy of predictions for welfare indicators based on routine herd data ranged from low to high. For many indicators, routine herd data can be used as a prescreening tool for detection of herds with poor welfare, and consequently reduce the number of farm visits needed for routine welfare assessments. Routine herd data also hold value for continuous monitoring of dairy cattle welfare.

http://dx.doi.org/10.3168/jds.2013-6585.

Prediction of insemination outcomes in Holstein dairy cattle using alternative machine learning algorithms. By Shahinfar et al., page 731. Prediction of the outcome of an insemination event can affect decision making on dairy farms. However, interactions between management and physiological features are very complex. Machine learning algorithms can be useful for understanding these complex interactions and developing tools that will help farmers make accurate reproductive management decisions.

http://dx.doi.org/10.3168/jds.2013-6693.

Optimizing production of in vivo-matured oocytes from superstimulated Holstein cows for in vitro production of embryos using $\mathrm{X}$-sorted sperm. By Matoba et al., page 743. The ability to produce calves of the desired sex is an attractive reproductive technology for dairy farmers wishing to breed replacement heifers. In vitro fertilization with in vivo-matured oocytes and sex-sorted sperm may be one way of maximizing the number of transferable female embryos in applied reproductive programs. Here, we report the establishment of an efficient method of producing female embryos from Holstein dairy cows by in vitro fertilization with $\mathrm{X}$-sorted sperm of in vivomatured oocytes collected by ovum pick up after superstimulation. Combined with dominant follicle ablation before superstimulation, large numbers of good-quality blastocysts can be obtained with this method.

http://dx.doi.org/10.3168/jds.2013-6838.

Effect of feed restriction on reproductive and metabolic hormones in dairy cows. By Ferraretto et al., page 754. Increasing feed intake can increase liver blood flow, potentially increasing metabolism of hormones such as progesterone (P4), leading to decreased circulating $\mathrm{P} 4$ concentrations. Lactating, pregnant cows had feed and energy restricted for $4 \mathrm{~d}$. As hypothesized, circulating insulin $(-33$ to $-63 \%)$ and milk production $(-11$ to $-40 \%)$ were reduced, whereas $\mathrm{P} 4(+8$ to $+24 \%)$ and nonesterified fatty acids ( +44 to $+414 \%)$ were increased. Thus, short-term feed restriction to $75 \%$ of normal intakes may be used in reproductive protocols to increase P4 and lower insulin concentrations. http://dx.doi.org/10.3168/jds.2013-6925.
Effects of acute feed restriction combined with targeted use of increasing luteinizing hormone content of follicle-stimulating hormone preparations on ovarian superstimulation, fertilization, and embryo quality in lactating dairy cows. By Bender et al., page 764. Metabolic and hormonal factors have the potential to affect ovarian superstimulatory success in cattle. We evaluated effects of reducing insulin concentrations (via feed restriction) as well as increasing luteinizing hormone (LH) content of folliclestimulatin hormone preparations on ovarian superstimulation, fertilization, and embryo quality. Fertilization and embryo quality were decreased in feed-restricted cows combined with low LH and in cows with ad libitum feeding combined with high $\mathrm{LH}$, indicating an interaction between LH stimulation and diet in oocyte and embryo quality. Thus, efforts to optimize superstimulatory protocols should consider the LH content of the superstimulatory preparation in relation to the nutritional program provided to the donor cow. http://dx.doi.org/10.3168/jds.2013-6926.

Effects of intramammary infusions of casein hydrolysate, ethylene glycol-bis( $\beta$-aminoethyl ether)-N,N, $\mathbf{N}^{\prime}, \mathrm{N}^{\prime}$-tetraacetic acid, and lactose at drying-off on mammary gland involution. $B y$ Ponchon et al., page 779. After cessation of milking, during involution, the mammary gland is highly susceptible to new intramammary infection. Once involution is completed, the gland becomes more resistant. The aim of this study was to determine whether intramammary infusions of casein hydrolysates, ethylene glycol-bis( $\beta$-aminoethyl ether)-N,N, $N^{\prime}, N^{\prime}$-tetraacetic acid (EGTA), or lactose solutions would hasten mammary gland involution. Intramammary infusion of casein hydrolysates was the most effective treatment to accelerate involution and may represent an opportunity to develop a nonantibiotic approach to increase mammary gland resistance to pathogens at drying-off.

http://dx.doi.org/10.3168/jds.2013-7062.

Physicochemical factors differentially affect the biomass and bacteriocin production by bovine Enterococcus mundtii CRL1656. By Espeche et al., page 789. Enterococcus mundtii CRL1656 from the bovine udder produces an inhibitory substance named mundticin CRL1656 that is active against bovine mastitis pathogens. The effect of physicochemical factors on Enterococcus mundtii CRL1656 biomass and mundticin CRL1656 production was studied. Optimal biomass and mundticin production were obtained in different conditions. Our results represent an advance in the optimization of culture conditions to obtain high levels of raw material (biomass and antimicrobial metabolite) that could be included in a probiotic product for bovine mastitis prevention.

http://dx.doi.org/10.3168/jds.2013-7070. 
Dairy cow handling facilities and the perception of Beef Quality Assurance on Colorado dairies. By Adams et al., page 798. Besides being a source of dairy products, the US dairy industry is a significant contributor to the beef supply. This study examined handling facilities currently used and practices carried out on the farm in order to ensure dairy beef quality. We observed a significant difference between preferred and actual location for intramuscular and subcutaneous injections. Moreover, beef quality assurance was not perceived as a priority when designing handling facilities. These results underscore the need for more effective educational interventions aimed at emphasizing the benefits of improved beef quality assurance in regard to dairy profitability and consumer confidence. http://dx.doi.org/10.3168/jds.2013-7146.

Comparison of peripartum metabolic status and postpartum health of Holstein and Montbéliarde-sired crossbred dairy cows. By Mendonça et al., page 805. Somatic cell count, incidence of metritis, and mortality have been reported to be greater in Holstein cows compared with crossbred cows. In the current experiment, Holstein cows tended to have a more pronounced decrease in dry matter intake in the last 15 $\mathrm{d}$ of gestation, but the lack of difference in concentrations of nonesterified fatty acids and $\beta$-hydroxybutyrate suggests that energy balance was not different between Holstein and crossbred cows. Holstein cows were more likely to develop at least one uterine disease postpartum compared with crossbred cows. The reduced incidence of uterine diseases in crossbred cows compared with Holstein cows could be a consequence of heterosis or breed complementarity.

http://dx.doi.org/10.3168/jds.2013-7159.

Bayesian validation of a serum and milk ELISA for antibodies against Mycobacterium avium subspecies paratuberculosis in Greek dairy goats across lactation. By Angelidou et al., page 819. Paratuberculosis, which is caused by Mycobacterium avium ssp. paratuberculosis, is a chronic intestinal infection in cattle, sheep, goats, and other ruminants and is responsible for substantial economic losses to the farming industry. We validated a commonly used diagnostic test, an ELISA, to detect the infection in paired milk and serum samples in dairy goats. The milk ELISA was as accurate as the serum ELISA. Because milk sampling is a noninvasive, rapid, and easy process, milk ELISA is preferred to the serum ELISA in dairy goats.

http://dx.doi.org/10.3168/jds.2013-7218.

The expression of genes involved in hepatic metabolism is altered by temporary changes to milking frequency. By Grala et al., page 838. The effects of short periods of once $(1 \times)$ - or thrice $(3 \times)$-daily milking immediately postpartum were investigated to determine if hepatic metabolism was affected during this metabolically challenging period. Relative to twice $(2 \times)$-daily milking, cows milked $1 \times$ had lower expression of genes involved in gluconeogenesis and ketogenesis and greater glucose concentrations, indicating improved energy status. When switched to $2 \times$ milking, cows previously milked $1 \times$ or $3 \times$ had greater gluconeogenesis relative to cows milked $2 \times$. Altering milking frequency changes hepatic metabolism in response to energy requirements for milk production.

http://dx.doi.org/10.3168/jds.2013-7321.

Consultancy to dairy farmers relating to animal health and herd health management on smalland medium-sized farms. By Pothmann et al., page 851. The objectives of this online survey were to obtain information about the challenges to farmers relating to animal health issues and the demands for consultancy by veterinarians or others regarding animal health and management on small- and medium-sized farms. Reproductive disorders, udder disease, lameness, and calf diarrhea were cited as the most pressing health problems. Besides performing practical work, veterinarians are important advisors in the fields of analysis of reproduction data, udder health, and animal welfare issues. The majority of the farmers requested better consultancy for fertility, feeding, data analysis, and sire selection.

http://dx.doi.org/10.3168/jds.2013-7364.

Effect of induced subclinical hypocalcemia on physiological responses and neutrophil function in dairy cows. By Martinez et al., page 874. Subclinical hypocalcemia $(\mathrm{SCH})$ was induced to determine the effects of low blood ionized Ca concentrations on physiological responses and function of immune cells in dairy cows. Induced SCH reduced feed intake and rumen contractions. Cows with induced SCH had reduced blood concentrations of insulin, increased concentrations of glucose and nonesterified fatty acids, and reduced cytosolic concentrations of $\mathrm{Ca}$ and function of neutrophils. Subclinical hypocalcemia compromises appetite, alters metabolism, and impairs function of immune cells in dairy cows.

http://dx.doi.org/10.3168/jds.2013-7408.

Short communication: Effects of analgesic use postcalving on cow welfare and production. By Stilwell et al., page 888. Giving an analgesic after calving improves the welfare of parturient dairy cows, increases feed intake, and may have financial advantages by allowing for higher milk production in primiparous animals.

http://dx.doi.org/10.3168/jds.2013-7100.

Short communication: Flooring preferences of dairy cows at calving. By Campler et al., page 892. 
Available knowledge on flooring and bedding preferences has been based mainly on research done with lactating cows. The aim of this study was to investigate if Holstein dairy cows have a flooring preference in the maternity pen. Preference for sand, rubber mats, or concrete, all bedded with a deep layer of straw, was determined using 17 cows. Cows showed an equal preference for calving on sand and concrete but rejected rubber mats. These results indicate that rubber mats are the least preferred by dairy cows in maternity pens. http://dx.doi.org/10.3168/jds.2013-7253.

Short communication: Maternal heat stress during the dry period alters postnatal whole-body insulin response of calves. By Tao et al., page $89 \%$. The effect of maternal heat stress (HT) during the dry period on insulin response of calves after weaning was evaluated. Calves were born to cows exposed to HT or cooling (CL) in late gestation. Compared with CL, HT calves had faster glucose clearance during glucose tolerance test and insulin challenge. Thus, maternal HT may enhance the whole-body insulin response of calves after weaning.

http://dx.doi.org/10.3168/jds.2013-7323.

Technical note: Selection of suitable reference genes for studying gene expression in milk somatic cell of yak (Bos grunniens) during the lactation cycle. By Bai et al., page 902. The suitability of 10 candidate reference genes as normalizers for quantitative analysis of gene expression was assessed in milk somatic cells isolated from the yak. The most stably expressed reference genes across lactation were RPS9, PPP1R11, UXT, and MRPL39. Use of a combination of these 4 genes is recommended for normalization in quantitative analysis of gene expression in milk somatic cells of lactating yak if similar experiments are performed.

http://dx.doi.org/10.3168/jds.2012-6437.

Effect of monensin in lactating dairy cow diets at 2 starch concentrations. By Akins et al., page 91\%. Feeding reduced-starch diets to lactating dairy cows has been reported to decrease feed efficiency, leading to the hypothesis that monensin may increase feed efficiency more for cows fed reduced-starch diets. The objective of this study was to determine the effects of monensin supplementation on lactation performance of dairy cows fed diets with either reduced or normal starch concentrations. Monensin improved lactation performance and feed efficiency at both concentrations of dietary starch. Feeding the reduced-starch diet reduced milk and protein yields, but feed efficiency was similar for both dietary starch concentrations. Dietary starch concentration and monensin supplementation had minimal effects on milk fatty acid composition. http://dx.doi.org/10.3168/jds.2013-6756.
Improved performance and heightened neutrophil responses during the neonatal and weaning periods among outdoor group-housed Holstein calves. By Cobb et al., page 930. The effects of single versus group outdoor housing on performance and leukocyte responses of dairy calves are not well known. Calves that were group housed had increased starter intake and average daily gain compared with calves housed singly. Similarly, neutrophil adhesion molecules and oxidative burst were greater in group-housed calves, which may be due to social interactions among groups. http://dx.doi.org/10.3168/jds.2013-6905.

The use of Lactobacillus species as starter culture for enhancing the quality of sugar cane silage. By Ávila et al., page 940. Sugar cane is a forage widely used in animal feed because of its high dry matter (DM) production and energy concentration. However, the ensilage of sugar cane has problems due to growth of yeasts, which might lead to DM losses. The use of a specific bacterial inoculant reduces the growth of yeasts and improves the silage quality. A novel screening process based on microbial metabolite and evaluation of the performance of these strains in experimental silos is proposed. The screening process was efficient in the selection of new inoculants, and the novel indigenous Lactobacillus hilgardii UFLA SIL51 and UFLA SIL52 strains were chosen for sugar cane silage. http://dx.doi.org/10.3168/jds.2013-6987.

Methane production, digestion, ruminal fermentation, nitrogen balance, and milk production of cows fed corn silage- or barley silage-based diets. By Benchaar et al., page 961. This study examined the effects of replacing barley silage with corn silage in the diet on enteric methane emissions and performance of dairy cows. Results showed that feeding cows diets with increasing amounts of corn silage $(0,27.2$, and $54.4 \%$ of dietary dry matter) increased dry matter intake, digestibility, and milk production. Methane production adjusted for gross energy intake declined with increasing dietary corn silage proportion, but the effect was more pronounced at the highest level of corn silage inclusion. Complete replacement of barley silage with corn silage in the diet could offer a strategy of reducing enteric methane emissions without negatively affecting dairy cow performance.

http://dx.doi.org/10.3168/jds.2013-7122.

Addition of potassium carbonate to continuous cultures of mixed ruminal bacteria shifts volatile fatty acids and daily production of biohydrogenation intermediates. By Jenkins et al., page 975. Diet-induced milk fat depression continues to have major economic impact in the dairy industry and is thought to occur from bacterial overproduction of lipid 
metabolites in the rumen, such as trans-10 18:1 and trans-10,cis-12 conjugated linoleic acid. Addition of potassium carbonate to fermenters reduced daily production of trans-10 18:1 and trans-10,cis-12 conjugated linoleic acid metabolites. Potassium carbonate is a possible dietary supplement to help alleviate economic losses from milk fat depression.

http://dx.doi.org/10.3168/jds.2013-7164.

Effects of feed additives on rumen and blood profiles during a starch and fructose challenge. By Golder et al., page 985. Feed additives perturb rumen function and may reduce the risk of ruminal acidosis. Four additives or combinations (virginiamycin, monensin plus tylosin, monensin plus yeast, or sodium bicarbonate plus magnesium oxide) compared with controls modified rumen function by apparently different mechanisms. No additive maintained completely favorable rumen conditions in all heifers when they underwent a substantial single challenge with grain and fructose. Considerable variation in rumen function was observed. Feeding behavior may be an important means by which cattle reduce risk of acidosis.

http://dx.doi.org/10.3168/jds.2013-7166.

Milk production and composition of mid-lactation cows consuming perennial ryegrass- and chicory-based diets. By Muir et al., page 1005. Grass herbage is the major component of dairy cow diets in temperate Australian dairy systems. However, forage herbs including chicory provide options to improve feed supply and nutrient intake over late spring, summer, and autumn. Feeding dairy cows chicory or mixed forage (chicory and perennial ryegrass) maintained milk production and did not negatively affect milk composition compared with perennial ryegrass alone, indicating that chicory or a mixed sward of chicory and perennial ryegrass could be used as an alternative forage to perennial ryegrass during spring.

http://dx.doi.org/10.3168/jds.2013-7183.

Effect of method of delivery of sodium butyrate on maturation of the small intestine in newborn calves. By Górka et al., page 1026. Dietary sodium butyrate stimulates small intestine development in calves. However, the optimal method of sodium butyrate delivery (milk replacer or starter mixture) for small intestine development stimulation has not been determined. Addition of sodium butyrate into milk replacer or starter mixture stimulated maturation of the small intestine and its supplementation in milk replacer had more pronounced effects. No synergistic effect of sodium butyrate addition into both milk replacer and starter mixture was observed.

http://dx.doi.org/10.3168/jds.2013-7251.
Oral administration of cobalt acetate alters milk fatty acid composition, consistent with an inhibition of stearoyl-coenzyme A desaturase in lactating ewes. By Frutos et al., page 1036. Previous investigations have shown that cobalt modifies milk fat composition in cattle but it remains unclear whether other ruminant species are also affected. Oral administration of cobalt acetate to lactating sheep altered milk fatty acid composition, consistent with an inhibition of stearoyl-coenzyme A desaturase. Indirect comparisons suggest that the effects of Co differ between sheep and cattle. Administration of Co has potential for the estimation of endogenous synthesis of fatty acids containing a cis-9 double bond in the mammary glands of lactating ruminants.

http://dx.doi.org/10.3168/jds.2013-7327.

Isoleucine, leucine, methionine, and threonine effects on mammalian target of rapamycin signaling in mammary tissue. By Arriola Apelo et al., page 104\%. Amino acids are substrates for protein synthesis. Specific amino acids are also known to regulate the rate of messenger RNA translation through the mammalian target of rapamycin (mTOR) pathway. In mammary tissue slices, Ile, Leu, and Thr activated the mTOR pathway, but showed antagonisms between them. Incorporation of the individual amino acid effects on translation regulation signaling into mammary protein synthesis models should improve milk protein predictions, which in turn would help to increase postabsorptive $\mathrm{N}$ efficiency and reduce $\mathrm{N}$ excretion by dairy cows.

http://dx.doi.org/10.3168/jds.2013-7348.

Compared with stearic acid, palmitic acid increased the yield of milk fat and improved feed efficiency across production level of cows. By Rico et al., page 105\%. We determined the effect of feeding palmitic and stearic acids at $2 \%$ of ration dry matter on the yield of milk and milk components, dry matter intake, and feed efficiency in cows with a wide range of milk production. Compared with stearic acid, palmitic acid increased milk fat concentration and yield, fat-corrected milk yield, and the efficiency of converting feed to milk. Thus, palmitic acid is more effective than stearic acid in increasing fat in milk. This finding may allow producers to better design diets and feeding strategies to improve milk component yields and farm profitability.

http://dx.doi.org/10.3168/jds.2013-7432.

Short communication: Effect of blackberry and pomegranate oils on vaccenic acid formation in a single-flow continuous culture fermentation system. By Ishlak et al., page 106\%. The effect of 
different oil sources on vaccenic acid formation in the rumen was studied using continuous culture fermenters. Three oil sources high in C18 unsaturated fatty acids (soybean, blackberry, and pomegranate oils) were each supplemented at $3 \%$ of diet dry matter. Vaccenic acid formation increased with all oil sources, particularly with blackberry oil. Pomegranate oil increased vaccenic acid formation mostly by lowering the reduction of vaccenic acid to stearic acid.

http://dx.doi.org/10.3168/jds.2013-6860.

Short communication: Effect of antioxidant supplementation on milk production, milk fat synthesis, and milk fatty acids in dairy cows when fed a diet designed to cause milk fat depression. By Boerman et al., page 1077. In this study, we investigated the effect of a synthetic antioxidant supplemented in a diet designed to cause milk fat depression on production variables and milk fatty acid composition. Diets high in unsaturated fats are known to cause lowered milk fat; however, finding ways to alleviate the negative effects of unsaturated fats will increase flexibility in ration formulation. The results provide information on the outcome of supplementing a synthetic antioxidant as well as the accompanying changes in biohydrogenation in the rumen. Milk fat concentration tended to increase, whereas milk protein concentration tended to decrease with antioxidant supplementation.

http://dx.doi.org/10.3168/jds.2013-7442.

Factors associated with age at slaughter and carcass weight, price, and value of dairy cull cows. By Bazzoli et al., page 1082. The value of cull cows at slaughter represents an important source of income for the dairy farm and is characterized by high variability. Factors associated with slaughter age, cull cow carcass weight, price per kilogram, and whole carcass value were quantified in dairy and dual-purpose cows. In mountain farms, Holstein-Friesian cows were younger at slaughter, yielded slightly lighter-weight carcasses, and received slightly lower prices and value than Brown Swiss cows. Dual-purpose cows were older, heavier, and received a greater price and total value at slaughter than dairy breeds, partly compensating for their lower milk productivity in Alpine dairy systems. http://dx.doi.org/10.3168/jds.2013-6578.

Economic values of production and functional traits, including residual feed intake, in Finnish milk production. By Hietala et al., page 1092. Improving feed efficiency of dairy cattle could have a substantial effect on economic efficiency and reduction of harmful environmental effects of dairy production through lower feeding costs and emissions from dairy farming. Therefore, improving feed efficiency should be considered for inclusion in the breeding goal for Nordic dairy cattle. To assess the economic importance of feed efficiency, the economic value of residual feed intake for Finnish Ayrshire cattle under production circumstances in 2011 was determined based on a bioeconomic approach, in which the profit of the production system was calculated using the generated steady-state herd structure.

http://dx.doi.org/10.3168/jds.2013-7085.

Assessment of accuracy of genomic prediction for French Lacaune dairy sheep. By Baloche et al., page 110\%. Lacaune dairy sheep are selected for milk production and udder and functional traits. Traditional male selection involves progeny testing young rams born from assortative mating. That method has been successful but is quite expensive. Recently, DNA information has become available early in the life of lambs. The accuracy of genomic selection based on best linear unbiased prediction (BLUP) methods and using DNA information from males at birth was studied, along with factors that affect accuracy. Genomic predictions are more accurate than pedigree-based predictions but less accurate in Lacaune dairy sheep than in Holstein dairy cattle.

http://dx.doi.org/10.3168/jds.2013-7135.

Using the unified relationship matrix adjusted by breed-wise allele frequencies in genomic evaluation of a multibreed population. By Makgahlela et al., page 1117. Most genomic evaluations in multibreed and admixed populations do not currently account for the differences in allele frequencies between breeds. As a result, the observed prediction accuracy is lower than that in single populations. Compared with ignoring the population structure, accounting for breed origin of alleles in the construction of the genomic relationship matrix tends to simplify the blending of genomic- and pedigree-based relationships for genomic evaluations. The validation reliabilities when accounting for breed composition, however, were low. http://dx.doi.org/10.3168/jds.2013-7167.

Effect of genetic merit for energy balance on luteal activity and subsequent reproductive performance in primiparous Holstein-Friesian cows. By von Leesen et al., page 1128. During early lactation, high-yielding dairy cows suffer from an energy deficit, which is known to affect fertility. In this study, daily breeding values for energy balance were estimated to describe the genetic merit for energy status in early and mid lactation. For a subset of cows with energy balance breeding values, progesterone measurements were available and first ovarian activity postpartum could be determined. The aim was to investigate the effect of genetic merit for energy balance on postpartum ovarian activity and subsequent reproductive performance. http://dx.doi.org/10.3168/jds.2013-7185. 
Fine mapping of a quantitative trait locus for bovine milk fat composition on Bos taurus autosome 19. By Bouwman et al., page 1139. This study reduced the region associated with fatty acids on bovine chromosome 19 to 2 candidate genes: $C C D C 57$ and FASN. The gene CCDC57 is minimally characterized and has not been associated with fatty acids previously but is expressed in the mammary gland. The gene FASN is known to be associated with fatty acids and is involved in de novo fat synthesis. Future studies using sequence data of both $C C D C 57$ and FASN, and eventually functional studies, will have to be pursued to assign the causal variants.

http://dx.doi.org/10.3168/jds.2013-7197.

Short communication: Genetic parameters of individual fatty acids in milk of Canadian Holsteins. By Bilal et al., page 1150. Genetic parameters of milk fatty acids (FA) in Canadian Holsteins were estimated. Most of the important milk FA displayed moderate heritability estimates, suggesting that milk fat composition of cows could be changed by genetic se- lection. Selection for monounsaturated FA or selection against saturated FA would seem to be an effective way of improving the FA composition of cow milk, although such a selection objective may somewhat reduce the present industry emphasis on selection for total fat. http://dx.doi.org/10.3168/jds.2012-6508.

Innovations in low input and organic dairy supply chains - What is acceptable in Europe? By Nicholas et al., page 115\%. Organic and low-input dairy systems contribute positive environmental benefits such as enhanced biodiversity and reduced pesticide and fertilizer use. By improving the competitiveness of these types of systems, more farmers will be encouraged to farm in this manner, maximizing the environmental returns to society and ensuring sustainable financial returns for farmers. Innovations in farming and supply chain practices can enhance the competitiveness of these systems, but only if everyone in the supply chain agrees on which innovations are acceptable to ensure maximum uptake of the innovation.

http://dx.doi.org/10.3168/jds.2013-7314. 